Grenzen nationaler Jurisdiktion sowie den Resolutionen zur Dritten Seerechtskonferenz (3. Abschnitt) und den Deklarationen zur Dritten Seerechtskonferenz der Vereinten Nationen (4. Abschnitt) dokumentieren die Herausgeber ausführlich die Entstehung und Entwicklung des Seerechtsübereinkommens bis zur Unterzeichnung des Vertragswerkes am 10. Dezember 1982.

In den verbleibenden Abschnitten der vorliegenden Textsammlung werden ergänzende, begleitende und für das Seerecht allgemein bedeutsame Dokumente abgedruckt.

So finden sich im fünften Abschnitt das Gesetz der Bundesrepublik Deutschland zur Regelung des Tiefseebergbaus vom 16. August 1980 sowie das Úbereinkommen vom 2. 9. 1982 über vorläufige Regelungen für polymetallische Knollen des Tiefseebodens. Der sechste Abschnitt enthält Rechtsakte der Bundesrepublik Deutschland betreffend ihr Hoheitsgebiet, wie etwa die Bekanntmachung der Bundesregierung vom 22. Januar 1964 über die Erforschung und Ausbeutung des deutschen Festlandsockels, während der letzte Abschnitt Rechtsakte anderer Staaten aufführt, wobei an dieser Stelle die berühmten Proklamationen des amerikanischen Präsidenten Truman zum Festlandsockel und zur Küstenfischerei vom 28. September 1945 als Beispiele zu nennen sind.

Insgesamt betrachtet haben Platzöder und Graf Vitzthum mit ihrer Textsammlung ein Werk vorgelegt, das in seiner Auswahl der Dokumente und in der Ubersichtlichkeit der Darstellung dieser Dokumente besticht. Jeder mit dem Völkerrecht befaßte Praktiker, Wissenschaftler oder Student, der diese Textsammlung auf seinem Schreibtisch hat, wird sicherlich dankbar sein, daß ihm die Suche nach den authentischen Texten der wichtigsten seerechtlichen Materialien erspart bleibt.

Andreas Buske

Lotte Müller-Ohlsen

Die Weltmetallwirtschaft im industriellen Entwicklungsprozeß

J. C. B. Mohr (Paul Siebeck), Tübingen, 1981, 265 S., DM 58,- br.

\title{
Roland Herrman
}

Exportinstabilität auf agrarischen Rohstoffmärkten. Situationsanalyse und Eingriffsmöglichkeiten

Kieler Wissenschaftsverlag Vauk, 1981, 292 S.

Josef Schraven

Internationale und supranationale Rohstofferwaltung

Duncker \& Humblot, Berlin, 1982, 193 S., DM 56,- 
Jamuna P. Agarwal/Hans H. Glismann/Peter Nunnenkamp

Olpreisschocks und wirtschaftliche Entwicklung. Anpassungsprobleme in der Dritten Welt

J. C. B. Mohr (Paul Siebeck), Tübingen, 1983, 261 S., DM 75,- br.

Bill Freund

Capital and Labour in the Nigerian Tin Mines

Longman, Harlow/Essex, 1981, 266 S., 17,5

\section{Ulrich Stürzinger}

Der Baumwollanbau im Tschad. Zur Problematik landwirtschaftlicher Exportproduktion in der Dritten Welt

Atlantis, Zürich, 1980, $266 \mathrm{~S}$.

\section{Peter Reinhard}

Bedeutung des Baumwollanbaus für die tschadische Landwirtschaft und Analyse der Geldflüsse, die durch ihn ausgelöst werden

Peter Lang, Bern, Frankfurt a. M., Las Vegas, 1981, 268 S. Sfr. 49,-

Die hier vorzustellenden Studien beschäftigen sich mit unterschiedlichem Erkenntnisinteresse und methodischem Zugang mit der Rolle und der Funktion der Rohstoff weltmärkte und der Rohstoffexportproduktion.

Die Kieler Ökonomin Müller-Ohlsen untersucht den Beitrag der NE-Metalle auf den Industrialisierungsprozeß auf makroökonomischer Ebene mit Daten und Zeitreihen, die z. T. bis in das 19. Jahrhundert zurückreichen. Sie behandelt die Produktionsstruktur, die Genese der modernen Metallwirtschaft durch industrielle Revolution und technischen Fortschritt, die Bestimmungsgründe für die Nachfrage, die Entwicklung des Verbrauchs, die Weltbergbau- und Raffinadeproduktion, den Welthandel und die Unternehmensstruktur, schließlich die Preisbildung und Preisentwicklung sowie die Regulierungsbestrebungen und die Entwicklungsperspektiven für die NE-Metalle, wobei sie die unterschiedlichen Eigenschaften und Entwicklungen der einzelnen Metalle kompetent herausarbeitet. Diese Studie wurde von der Metallgesellschaft angeregt und finanziell gefördert. Ihr Erkenntnisinteresse wird daher primär durch die Sicht der Industrieländer bestimmt, obwohl durchaus - aus "entwicklungspolitischen Gründen" etwa für den Ausbau der Hüttenkapazitäten in den Bergbauländern plädiert wird (S. 123), ohne daß dies allerdings vertieft würde. Das hier aufbereitete Material und die Datenzusammenstellungen sind dennoch für den Entwicklungsländerforscher von Interesse, so u. a. besonders die Beschreibung des Konzentrationsprozesses unter den Bergbauunternehmen, die Preisbestimmung und Preisentwicklung (seit 1890), der Umfang und die Rolle des Recycling der Metalle, die historisch in der Mehrzahl von den europäischen/amerikanischen Unternehmen bzw. Staaten versuchten Kartellierungsund Regulierungsbemühungen u. v. a. m. Auf die Probleme und die Problematik der 
Metallexporte für die Entwicklungsländer wird in diesem Buch nicht eingegangen, das war offenbar auch nicht dessen Aufgabe.

Mit einem für die rohstoffexportierenden Entwicklungsländer wichtigen Einzelaspekt der Exportinstabilität - befaßt sich hingegen die Kieler Dissertation des Agrarökonomen Roland Herrman. Er beschäftigt sich mit der Frage, ob die Exportinstabilität das Wachstum in den Entwicklungsländern hemmt oder doch gar positiv verursacht, wie inzwischen auch einige Autoren (z. B. Knudsen/Parnes) behaupten und unterzieht die inzwischen recht umfangreiche Literatur zu diesem Thema einer kompetenten methodenkritischen Diskussion. Anschließend untersucht Herrman mit ökonometrischen Methoden die Gründe für die Import- und Exportniveauschwankungen und Exporterlösschwankungen für zahlreiche Agrarprodukte sowie die Ursachen und Konsequenzen der Preisschwankungen des Kaffee- und Kakaomarktes auf die Erlöse der einzelnen Produzentenländer, da beide Märkte durch überdurchschnittliche Exportmengeninstabilitäten charakterisiert sind. Unter den Regulierungsmöglichkeiten streicht er die größere Eignung des Stabex-Systems der EG bzw. auch die geplante UNCTAD-Erlösstabilisierung gegenüber Preisstabilisierungspolitiken heraus, da dadurch die nationalen Unterschiede im Stabilisierungsbedarf besser beachtet werden können. Andererseits macht er jedoch auch deutlich, daß durch die (geplante) Bruttokompensation der Exportausfälle durch die UNCTAD und durch das Stabex-System, das er für die Jahre 1975-79 untersucht, eigentlich keine Erlösstabilisierung stattfindet, sondern nur Erlösausfälle kompensiert werden. Erlösausfälle können nämlich nur ex post ante berechnet und (meist noch viel später) ausgezahlt werden. Stabex ist daher für Herrman mehr ein Versicherungssystem, wobei die Zahlungsströme überwiegend Geschenke sind bzw. Geschenkcharakter haben, deren Verteilung allerdings auch nicht zielgerichtet rational erfolgt.

Ausführlicher als Herrman beschäftigt sich Josef Schraven in seiner in Speyer eingereichten juristischen Dissertation mit den Rohstoffregulierungsversuchen. Die Arbeit bietet in ihren beiden ersten Teilen einen brauchbaren Uberblick über die zahlreichen internationalen Regulierungsbemühungen durch Anbieter bzw. Produzenten- und Käuferzusammenschlüsse (beginnend mit den Zuckerkonventionen des 19. Jahrhunderts), ohne dabei allerdings vollständig zu sein. Daran schließt sich eine vergleichende Betrachtung der Lenkungsformen und Organe der zwischenstaatlichen Rohstoffverwaltungen an. Im letzten Teil behandelt Raven die überstaatliche Verwaltung in der gelenkten Rohstoffwirtschaft Westeuropas, d. h. insbesondere den Agrarmarkt der E(W)G. Dieser Teil ist weitgehend rechtsdogmatisch - unter Vernachlässigung der Praxis - geschrieben. Den EWG-Strukturen wird eine höhere Komplexität ihrer Verwaltungsstrukturen und -verfahren bescheinigt. Schraven stellt aber abschließend etwas zu ahnungslos die Frage: "Wie weit sich die supranationale Fortentwicklungen für die zwischenstaatlichen Verwaltungseinheiten verwerten lassen, bleibt abzuwarten." Eigentlich liegt hier einige - gar nicht so positive - Erfahrung schon vor!

Immerhin gelang es bisher in der EG die Agrarpreise einigermaßen zu stabilisieren, wenn auch unter erheblichen Kosten für Verbraucher und Steuerzahler, die die politi- 
schen Instanzen bisher auch aufzubringen bereit waren. Zu dieser weitreichenden Bereitschaft zur Regulierung der Preise waren weder Produzenten- noch Konsumentenländer in den übrigen Rohstoffsektoren bereit. Entsprechend selten sind auch die Erfolge, durch internationale Aktionen die Rohstoffpreise administrativ zu stabilisieren. Auch der Olmarkt macht hier keine grundsätzliche Ausnahme. Viele Jahre und Jahrzehnte waren hier sinkende Nominal- und insbesondere Realpreise zu verzeichnen. Lediglich 1973/74 und 1979/80 vermochten die Preise drastisch heraufgesetzt zu werden. Diese bröckelten real zwar anschließend immer wieder ab, blieben aber auf dem jeweils erreichten hohen Niveau. Die im Kieler Institut für Weltwirtschaft angef ertigte Studie von Agarwal/Glisman/Nunnenkamp beschäftigt sich ausführlich mit dem Einfluß der ersten und etwas knapper und vorläufiger mit den Auswirkungen der zweiten Olpreiserhöhung auf die Wirtschaft der Nicht-Öl-Entwicklungsländer (nennen wir sie: NichtOPEC-Entwicklungsländer oder NOPEC).

Die Kieler Autoren gehen sehr abgewogen und differenziert dem Ausmaß des Anstiegs der Olimportrechnung, der Entwicklung der Exporte als Folge verstärkter Nachfrage der OPEC-Länder und aufgrund der Nachfrageschwäche der Industrieländer, der Entwicklung der realen Austauschverhältnisse, den Veränderungen der internationalen Wettbewerbsfähigkeit, der Entwicklung der gesamtwirtschaftlichen Produktion und dem Zusammenhang von Ölpreis und Inflation nach. In einem zweiten Teil untersuchen sie die wirtschaftspolitischen Reaktionen der NOPEC-Staaten und gehen dabei auf Einfuhrbeschränkungen, exportfördernde, wechselkurspolitische und geld- und fiskalpolitische Maßnahmen ein. Im dritten Teil werden diese Fragen etwas knapper für den zweiten Olpreisschock behandelt. Trotz einer Verfünffachung der Olimportrechnungen der NOPEC sind diese nicht vorrangig, nach Agarwal et. al., für die Verschlechterung der Handelsbilanzen dieser Länder verantwortlich. Wichtig war vor allem auch der Nachfrageausfall der Industrieländer, den die Autoren zur Hälfte auf ölpreisbedingte Sozialproduktverluste zurückführen. Insgesamt sollen aber die Sozialprodukteinbußen in den NOPEC-Staaten aufgrund der Ölpreissteigerungen gering geblieben sein. Durch eine erhöhte Verschuldung mußten allerdings die Leistungsbilanzdefizite, die in einigen Fällen durch Import-,Export- und Wechselkurspolitik aufgefangen werden konnten, abgedeckt werden. Auch für die Auswirkungen des zweiten Olpreisschocks kommen die Autoren zu keinem grundsätzlich anderen Ergebnis. Erst die Verschlechterung der allgemeinen weltwirtschaftlichen Rahmenbedingungen seit 1981 führte auch zu einer drastischen Verschärfung der Lage in den meisten NOPEC-Staaten.

Es war nicht Gegenstand der besprochenen Kieler Studie, die Auswirkungen der Olpreishaussen auf die Förder- und Exportländer selbst zu untersuchen. Nigeria ist ein solches Ol- und OPEC-Land. Im Ausklang seiner sozial- und wirtschaftshistorischen Studie über den Zinnbergbau in diesem Land geht der britische Marxist Bill Freund auch auf die Einflüsse des (ersten) Olbooms auf diesen traditionalen Exportsektor ein: Die durch den Olpreis- und Ölrentenboom induzierte hektische Bautätigkeit im Lande, die Anheizung der Inflation, die Verknappung der Arbeitskräfte und die Verteuerung der Lohnkosten, verbunden mit einer zunehmenden Erschöpfung der Reserven, führte nahezu zu 
einem Zusammenbruch dieses Wirtschaftszweiges. Nicht viel anders ging es einigen agrarischen Exportsektoren (Erdnüsse, Kakao). Es läßt sich erahnen, in welche Schwierigkeiten Nigeria geraten mußte, als die Olrenten wieder drastisch reduziert wurden (seit 1981). Damit wird allerdings nur das absehbare Ende dieses Rohstoffexportsektors markiert. Bill Freund zeichnet in seiner detaillierten Studie die Entwicklung dieses Sektors seit vorkolonialer Zeit nach und stützt sich dabei auf die Auswertung eines umfangreichen Archivmaterials in Nigeria und England. Er zeigt, daß der Zinnbergbau schon vor der britischen Eroberung ein wichtiger Wirtschaftszweig in ZentralNigeria war, daß die Übernahme durch das europäische Handelskapital zunächst mit keinen technologischen Neuerungen verbunden war, welche Schwierigkeiten es zunächst gab, Arbeitskräfte zu rekrutieren und welche historische Entwicklung die gesellschaftliche Organisation des Zinnbergbaus in Nigeria in den verschiedenen Phasen bis in die Gegenwart (seine Daten reichen bis 1976) genommen hat, welche Gewinne hier - wann erzielt werden konnten, wie die Arbeitsverhältnisse, die Arbeitsbedingungen und die Löhne sich entwickelt haben. Dabei gelingt ihm eine sinnvolle Verknüpfung der generellen Weltmarktentwicklung mit den Ereignissen auf dem Jos-Plateau in Nigeria. Es wird deutlich, daß dieser Rohstoffexportsektor durchaus nicht zum Nutzen aller Beteiligten ausgebeutet wurde, daß durch die koloniale Extraktionspolitik in mancher Hinsicht sogar eine Regression stattfand (etwa durch Aufgabe der Zinnverhüttung, die erst in den 60er Jahren wieder aufgenommen wurde), daß die kapitalistischen Unternehmen den Wert der Arbeitskraft durch Konskriptionen, Zwangsarbeit, zunehmend auch durch ökonomischen Zwang zu drücken vermochten, langanhaltende depressive Weltmarktpreise immer auf Kosten der Löhne und der Lohnsumme ging, während die Gewinne u. U. noch auf einer passablen Höhe gehalten werden konnten. Freund lief ert allerdings auch das Material für durchaus relevante Veränderungen etwa seit dem Zweiten Weltkrieg: Gewerkschaftliche Organisation und Agitation, die Nigerianisierung der Politik, die zur Selbstverwaltung und schließlich völkerrechtlichen Unabhängigkeit des Landes führte, erzwangen einen kontinuierlichen Anstieg der Reallöhne, der erst durch die hohe Inflation in den 70er Jahren unterbrochen wurde, sowie eine ganz erhebliche Steigerung des (nigerianischen) Steueranteils am Produktionswert, außerdem die (Wieder)Aufnahme der Zinnverhüttung, all dies bei einer deutlichen Absenkung der Gewinnrate der kapitalistischen Zinnproduktion und damit allerdings, auch bedingt durch den Rückgang der Arbeitsproduktivität und unzureichenden Investitionen, ein allgemeiner Rückgang des Produktionsvolumens.

Bill Freund vermag darin allerdings keine positiven Aspekte zu erblicken. Der Auf- und Ausbau der Sozialeinrichtungen für die Zinnarbeiter sind für ihn nur "Wohlfahrtsfacaden" (S. 218). Für ihn bedeutet die Nigerianisierung noch nichts, da sie hier nur neue Klassenverhältnisse begründe und im übrigen die Mehrwertaneignung nicht grundsätzlich verändere: "The nationalist politicians sought to divert capital in their own interests as a potential capitalist class: they squabbled over which clique, party and region would get the best share in the surplus. However, they did not try to alter the way in which surplus was extracted from labour in Nigeria." (S. 219). Das ist zwar nicht falsch, 
negiert allerdings die vorhandenen Fortschritte zugunsten einer umfassenderen und radikaleren Lösung, über deren Definition der Autor den Leser im Unklaren läßt.

Mit der Problematik der - in diesem Falle - agrarischen Exportproduktion, des Baumwollbaus im Tschad, befassen sich zwei Schweizer Wissenschaftler in ihren Dissertationen. Der Historiker Ulrich Stürzinger verfolgt, auf der Basis von im wesentlichen in Europa gesichteten Archiv- und Sekundärmaterials sowie eines kurzen Informationsaufenthaltes im Tschad (1978), kritisch die zwangsweise Einführung des Baumwollanbaus im Tschad (in den 20er Jahren). Er behandelt ihre flächenmäßige Ausdehnung, ihre Bedeutung für Exporte und Staatshaushalt, betrachtet die Instrumente zu ihrer Förderung, die Einkommensentwicklung der Bauern. Bei all dem macht er die gesellschaftlichen Interessen deutlich, die in Frankreich und schließlich auch im Tschad, nach der völkerrechtlichen Unabhängigkeit, die Formierung dieses Exportsektors betrieben haben, der als (untaugliches) Mittel der "Modernisierung" bisher nicht seine politische Attraktivität verloren hat. Nicht zuletzt aufgrund der (verkehrs)geographischen Ungunstlage war und ist eine rentable Weltmarktproduktion von Anfang an nur durch staatliche französische Subventionen möglich, an deren Stelle inzwischen der EG-Entwicklungsfonds getreten ist. Da Baumwolle den Bauern nur ein bescheidenes Geldeinkommen, das real sogar in den letzten Jahren gesunken ist, ermöglicht, dabei in Konkurrenz zum Nahrungsmittelanbau steht und - durch Verkürzung der Brachezeit zur Gefährdung der Lebensgrundlage der Menschen, durch zunehmende Bodenverschlechterung, beiträgt, charakterisiert Stürzinger den Entwicklungsweg über die Baumwolle als eine Sackgasse. Sollte man an diesem festhalten, würde dies in eine landwirtschaftliche und menschliche Katastrophe führen.

Er fordert stattdessen die Förderung der "Gemeinschaftsbildung" und "Verselbständigung", da Entwicklung ohne "Eigeninitiative" nicht denkbar erscheine. Das sind allerdings etwas unverbindliche Worthülsen, wie sie engagierten kritischen Autoren an europäischen Schreibtischen immer wieder durch die Feder fließen. Eine "Entwicklung" des Tschad, im Sinne einer Verbreiterung und Verbesserung der Lebensgrundlagen für seine Bevölkerung, hat in jeder Richtung gewaltige, möglicherweise überhaupt nicht lösbare, Probleme zu überwinden. Allein aufgrund der Binnenlage und den begrenzten natürlichen Ressourcen - vom permanenten Bürgerkrieg ganz zu schweigen - ist das Entwickungspotential als nicht sehr ausbaufähig einzuschätzen. Gewiß ist es ein zu korrigierender Fehler - aus entwicklungspolitischer Sicht - daß die Nahrungsmittelproduktion in so vieler Hinsicht zugunsten der Baumwolle bei staatlichen Förderungsmaßnahmen benachteiligt wird, so daß inzwischen immer mehr Nahrungsmittel eingeführt werden müssen, die ganz gut wohl auch im Lande hätten erzeugt werden können. Zucker und Tee, inzwischen "Luxuskonsumgüter" auch der ärmeren Marktbauern, müßten aber in jedem Fall importiert werden, oder auch nicht? Autozentrierte Entwicklung nur auf der vorhandenen Ressourcenbasis wäre, konsequent durchgeführt, allenfalls Stagnation, wahrscheinlich - nicht zuletzt aufgrund des Bevölkerungswachstums Regression. Also, es muß sehr bezweif elt werden, ob der Tschad wirklich ganz auf die Baumwollexportproduktion verzichten sollte und könnte, immerhin so lange eine be- 
triebswirtschaftliche und volkswirtschaftliche Rentabilität durch ausländische Subventionen sichergestellt werden kann. Das bedeutet jedoch nicht, wie Stürzinger plausibel aufzeigt, daß man den Baumwollanbau immer weiter ausdehnen kann, ihn zum führenden Entwicklungsmotor machen sollte. So lange Bodenerosion und Bodenauslaugung durch Verkürzung der Brachezeit im vorherrschenden Wanderfeldbau nicht aufgehalten werden kann, muß dies eben die beschriebenen und prognostizierten negativen Langzeitwirkungen haben.

Aufschlußreich in dieser Hinsicht ist die Untersuchung des Agrarökonomen Peter Reinhard zum selben Thema. Während aber im Mittelpunkt der Arbeit von Stürzinger die historisch-genetische Entwicklung des Baumwollanbaus und deren Makroentwicklung steht, hat Reinhard Daten auf Mikroebene gesammelt und ausgewertet. Methodisch ist dieser Ansatz also erheblich anspruchsvoller und aufwendiger, gepflastert aber auch mit zahlreichen Problemen, die nicht alle ausgeräumt werden konnten (wie der Autor z. T. selbst kritisch offenlegt).

Reinhard hat 450 Fragebögen an Baumwollbauern verteilt, in denen diese ihren Betriebsaufwand und Betriebsergebnisse, ihre Einnahmen und Ausgaben für zwei Betriebsjahre notieren sollten (die Ausweitung des Bürgerkrieges führte allerdings zum Abbruch der Untersuchung nach einem Jahr 1977/78). 180 auswertbare Bögen - darunter allerdings nur 125 für den monetären Bereich - hat Reinhard zurückbekommen. Auf dieser - relativ kleinen - Basis beruht im wesentlichen seine vorliegende Untersuchung. Nach den Daten Reinhard's machen die Baumwollverkäufe im Durchschnitt etwa $40 \%$ der monetären Einkommen der Untersuchungsbetriebe aus, jeweils ein knappes Drittel wird durch andere betriebliche sowie durch außerbetriebliche Einnahmen erzielt. Hinter diesen Durchschnittszahlen verbergen sich erhebliche Schwankungen, nicht zuletzt aufgrund unterschiedlich großer Baumwollanbauflächen und Flächenproduktivitäten. Die große Bedeutung, die man der Baumwolle für die betrieblichen monetären Einkommen beimißt, hat sie damit offenbar nicht. Dies wird auch deutlich, wenn man die monetären Ausgaben betrachtet. Reinhard schätzt, daß allenfalls $2 / 3$ der monetären Ausgaben für lebensnotwendige Güter aufgewendet werden, etwa $40 \%$ könnte allerdings auch nichtmonetär beschafft (und gehandelt) werden. Da die Arbeitsverdienste mit der (arbeitsintensiven) Baumwolle recht niedrig liegen, kann die Ausweitung ihres Anbaus weithin nur durch den staatlichen Anbauzwang (der, nach Reinhard, 1975 aufgehoben wurde, aber faktisch 1977/78 noch nicht überwunden war) und durch die auf die Baumwolle konzentrierte Infrastruktur sowie die Subventionen (der Inputs) erklärt werden. Reinhard macht deutlich, wie sowohl (gegenwärtig) die EG, die die Kosten der Inputs bis zu $50 \%$ subventioniert, als auch der Staatsapparat des Tschad von der Expansion des Baumwollanbaus profitieren: Erstere durch Steigerung der Exporte des Tschad, die auch Importe - in erster Linie aus der EG - ermöglichen; letzterer durch Steigerung der Staatseinnahmen, die allerdings in der Nettobilanz nur möglich sind, da die produktivitätssteigernden Subventionen nicht vom Staat selbst, sondern eben von der EG bezahlt werden. Die Konsequenz ist, daß die Baumwolle auch dort angebaut wird, wo die betriebswirtschaftlich (ohne Subventionen) und volkswirtschaftlich, durch Degradation 
der Böden, nicht rentabel ist. Reinhard's Plädoyer für eine völlige Liberalisierung der Anbauentscheidungen der Bauern, eine Erhöhung der Produzentenpreise auf Kosten des Wegfalls der Inputsubventionen und einer gleichmäßigen staatlichen Förderung der Nahrungsmittelproduktion ist voll zuzustimmen. Unter den gegebenen Verhältnissen im Tschad kann die Baumwolle nicht "Motor der Entwicklung" sein. Ganz ersetzen wird man sie aber wohl auch nicht können.

Rolf Hanisch

Hans A. Havemann/Hussein M. Rady

Technologiehilfe für die Dritte Welt: Die Evolution der Entwicklungstechnik. Systematik - Forschung - Beratung - Beispiele

Internationale Kooperation (Aachener Studien zur internationalen technisch-wirtschaftlichen Zusammenarbeit), Band 20, Nomos Verlagsgesellschaft, Baden-Baden, 1979, 616 S., DM 84,-

Im Zentrum der Betrachtung steht hier das Plädoyer für eine Technik, die aus den besonderen Gegebenheiten der Umwelt abgeleitet ist, den gesamtgesellschaftlichen Bedürfnissen von Entwicklungsländern entspricht und einen Einfluß auf ihre allgemeinen Wachstumslinien ausübt. Eine "situationskonforme Entwicklungstechnik" bestünde in der optimalen Anpassung der aus westlichen Industrieländern exportierten technischen Güter an Gesellschaften der Dritten Welt.

Es ist nur konsequent, daß die Autoren dabei eine Entwicklungsstrategie im Vesier haben, die von der heute im allgemeinen praktizierten abweicht, da sie vorrangig Ziele wie Arbeitsplatzbeschaffung, egalitäre Wohlstandserhöhung, Schonung der Umwelt und Steigerung der Produktion zur Befriedigung lokaler Grundbedürfnisse verfolgt. Anhand verschiedener Beispiele werden die Nachteile der bisher üblichen technologieintensiven Großprojekte aufgezeigt. Am Assuan-Hochstaudamm z. B., der mit sowjetischer Technologie- und Kapitalhilfe gebaut wurde, zeigen die Verf. die teilweise katastrophalen Folgen solcher milieufremden Importtechnologie (z. B. Schäden durch das Ausbleiben der saisonmäßigen Schwankung des Grundwasserspiegels für die Landwirtschaft der Fellachen; Erosion der Delta-Küste; Zunahme von Versalzung, Schädlingen etc.).

Andererseits werden alternative Technologieprojekte vorgestellt, z. B. Aufforstungsprojekte in ariden und semi-ariden Gebieten, in denen nicht geschlossene Grüngürtel über viele Kilometer zu immensen Kosten angelegt werden (wie z. B. in Algerien oder beim GTZ-Projekt im Süd-Sudan!), sondern bei denen zahlreiche kleinere Grünzonen um die ruralen Siedlungszentren herum angelegt werden - auch um den lokalen Bedarf an Brenn- und Bauholz befriedigen zu können.

Interessant sind auch die Alternativvorschläge zur besseren Nahrungsmittelversorgung 